OHSTPY-HEP-T-98

hep-ph/9804280

November 1, 2018

\title{
Dimensional Reduction of the Two Higgs Doublet Model at High Temperature
}

\author{
Jens O. Andersen \\ Department of Physics, The Ohio State University, Columbus, OH 43210
}

\begin{abstract}
Dimensional reduction and effective field theory methods are applied to the Two Higgs Doublet Model at finite temperature. A sequence of two effective three-dimensional field theories which are valid on successively longer distance scales is constructed. The resulting Lagrangian can be used to study different aspects of the phase transition in this model as well as the sphaleron rate immediately after the phase transition.
\end{abstract}




\section{Introduction}

The electroweak phase transition (EWPT) has been the subject of intense investigation in recent years, largely due to its possible role in generating the baryon asymmetry of the present Universe [12] (see also [3] for a detailed review). If the electroweak phase transition is of first order, it proceeds through bubble nucleation, and the baryon asymmetry is produced as the bubbles expand and the Universe is far from equilibrium [1-2]. Moreover, if the baryon asymmetry produced during the phase transition has survived until today, baryon number violating processes (sphaleron processes) must have been suppressed immediately after the phase transition [4]. This requires that the electroweak phase transition is strongly first order [4]. It is now well established that this requirement is not met in the Standard Model (SM); for realistic values of the Higgs mass, the phase transition is either too weakly first order to suppress the sphaleron processes, or it is second order, or there is no phase transition at all [5]. Furthermore, it is not clear that the amount of $\mathrm{CP}$ violation in the $\mathrm{SM}$ is sufficient.

The fact that baryogensis is ruled out in the Standard Model suggests the investigation of extensions of the Standard Model such as the Minimal Supersymmetric Standard Model (MSSM) and the Two Higgs Doublet Model (2HDM) [6]. Both these theories have additional sources of $\mathrm{CP}$ violation. The main objective is to find regions in parameter space where the phase transition is strongly first order, so that the excess of baryons produced during the phase transition is not washed out by sphaleron processes immediately after the phase transition.

The electroweak phase transition in the Standard Model [7-9] as well as in the Two Higgs Doublet Model [10-14] has been studied using resummed perturbation theory. The strength of the EWPT in the Standard Model weakens as the Higgs mass increases [7-9]. However, the resummed loop expansion breaks down for large (realistic) Higgs masses (see e.g. Ref. [8] for a discussion of the validity of the resummed perturbation expansion), and so one must employ nonperturbative methods in order to discriminate between a weakly first order and a second order phase transition.

The electroweak phase transition in the SM has also been investigated by lattice simulations directly in four dimensions [15-17], renormalization group techniques [18-19] and the $\epsilon$-expansion [20]. These methods yield results for the quantities characterizing the phase transition that are in qualitative agreement with the perturbative treatment.

Significant progress in the study of phase transitions has been made by applying the methods of dimensional reduction [21-24] and effective field theory [25]. The idea is to integrate out the nonzero bosonic modes as well as the fermionic modes which decouple from the static modes at high temperature [21-24]. One is then left with an effective three-dimensional field theory of the zero modes. Since the nonstatic modes have masses of order $T$, the process of dimensional reduction is free of infrared problems, and in weakly coupled theories this can normally be carried out perturbatively. In nonabelian gauge theories the effective three-dimensional theory contains two momentum scales [26]. The scale $g T$ is provided by the temporal component of the gauge field, and 
the scale $g^{2} T$ is provided by the spatial components of the gauge field. Moreover, in theories with a single Higgs multiplet, the scalar mass is normally of order $g^{2} T$ for temperatures close to $T_{c}$. For theories with more than one Higgs multiplet, the masses of the additional scalar fields are generally of order $g T$. In either case it proves useful to construct a second effective field theory by integrating out the timelike component of the gauge field (and possibly some scalar fields) as shown by Farakos et al. 27] and by Braaten and Nieto [28]. This approach has made effective field theory a very powerful tool for studying field theories at high temperatures. Perturbation theory breaks down for the resulting effective theory close to the phase transition and it is also severely infrared divergent in the symmetric phase. So one must use nonperturbative methods such as lattice simulations to investigate the phase transition. Dimensional reduction has been applied to a number of theories with spontaneously broken gauge theories: $S U(2)+$ Higgs [27,29-30], the Standard Model [31], the MSSM [32-36], $S U(5)+$ Higgs [37], the 2HDM [34] and $U(1)+$ Higgs [27,38-39]. The threedimensional effective theories have been studied numerically in e.g. Ref, [40-44] for $S U(2)+$ Higgs, in Ref. 45] in the case of $S U(2) \times U(1)+$ Higgs, in Refs. [39,46-53] for $U(1)+$ Higgs, and in Ref. [54] for $S U(3) \times S U(2)$ with two scalar fields (The latter arises as an effective $3 d$ theory for MSSM for some values of the parameters).

In the present paper we reconsider the Two Higgs Doublet Model. The model is interesting in its own right, but the $2 \mathrm{HDM}$ (with the temporal component of the gauge field as an additional adjoint Higgs field) in $3 d$ also arises as an effective theory for the MSSM [31]. In Ref. [34] dimensional reduction for this model was carried out in the one-loop approximation. The second effective theory obtained by integrating out the timelike component of the gauge field was constructed in Refs. [32, 34, also at the one-loop level. In order to obtain a satisfactory accuracy for the thermodynamic quantities describing the phase transition, the scalar mass parameters in the $3 d$ theory are needed to two-loop order [31]. This calculation is carried out in the present paper.

The plan of the article is as follows. In section II we briefly discuss the Lagrangian for the Two Higgs Doublet Model. In section III we present the parameters of the first effective theory. Section IV is devoted to the scenario where one of the Higgs doublets is heavy, and is integrated out together with the timelike component of the gauge fields. In Section $\mathrm{V}$ we consider the case where both Higgs doublets are light and are retained in the final effective Lagrangian. Finally, in section VI we summarize. In Appendix A, the notation and conventions are given. We also list the necessary sum-integrals in the underlying theory as well as the three-dimensional integrals needed in the effective theory. In Appendix B, some details of the matching procedure are given by explicitly calculating a mass parameter in the first effective theory. 


\section{Two Higgs Doublet Model}

The Euclidean Lagrangian for the $S U(2)$ gauge-invariant 2HDM without fermions reads

$$
\begin{aligned}
\mathcal{L}= & \frac{1}{4} G_{\mu \nu} G_{\mu \nu}+\left(D_{\mu} \Phi_{1}\right)^{\dagger}\left(D_{\mu} \Phi_{1}\right)+\left(D_{\mu} \Phi_{2}\right)^{\dagger}\left(D_{\mu} \Phi_{2}\right)+m_{1}^{2} \Phi_{1}^{\dagger} \Phi_{1}+m_{2}^{2} \Phi_{2}^{\dagger} \Phi_{2} \\
& +m_{3}^{2}\left(\Phi_{1}^{\dagger} \Phi_{2}+\Phi_{2}^{\dagger} \Phi_{1}\right)+V\left(\Phi_{1}, \Phi_{2}\right) .
\end{aligned}
$$

Here, $\Phi_{1}$ and $\Phi_{2}$ are the $S U(2)$-doublets

$$
\Phi_{1}=\frac{1}{\sqrt{2}}\left(\begin{array}{c}
\phi_{1}+i \eta_{1} \\
\phi_{2}+i \eta_{2}
\end{array}\right), \quad \Phi_{2}=\frac{1}{\sqrt{2}}\left(\begin{array}{c}
\phi_{3}+i \eta_{3} \\
\phi_{4}+i \eta_{4}
\end{array}\right)
$$

and

$$
D_{\mu} \Phi_{i}=\left(\partial_{\mu}-i g \tau^{a} A_{\mu}^{a} / 2\right) \Phi_{i}, \quad G_{\mu \nu}^{a}=\partial_{\mu} A_{\nu}^{a}-\partial_{\nu} A_{\mu}^{a}+g \epsilon^{a b c} A_{\mu}^{b} A_{\nu}^{c}
$$

Here, $g$ is the gauge coupling, $i=1,2$ and $\tau^{1}, \tau^{2}$ and $\tau^{3}$ are the three Pauli matrices.

The potential $V\left(\Phi_{1}, \Phi_{2}\right)$ is [5, 32, 34]

$$
\begin{aligned}
V\left(\Phi_{1}, \Phi_{2}\right)= & \lambda_{1}\left(\Phi_{1}^{\dagger} \Phi_{1}\right)^{2}+\lambda_{2}\left(\Phi_{2}^{\dagger} \Phi_{2}\right)^{2}+\lambda_{3}\left(\Phi_{1}^{\dagger} \Phi_{1}\right)\left(\Phi_{2}^{\dagger} \Phi_{2}\right)+\lambda_{4}\left(\Phi_{1}^{\dagger} \Phi_{2}\right)\left(\Phi_{2}^{\dagger} \Phi_{1}\right) \\
& +\lambda_{5}\left[\left(\Phi_{1}^{\dagger} \Phi_{2}\right)\left(\Phi_{1}^{\dagger} \Phi_{2}\right)+\left(\Phi_{2}^{\dagger} \Phi_{1}\right)\left(\Phi_{2}^{\dagger} \Phi_{1}\right)\right]+\lambda_{6}\left[\left(\Phi_{1}^{\dagger} \Phi_{1}\right)\left(\Phi_{2}^{\dagger} \Phi_{1}\right)+\left(\Phi_{1}^{\dagger} \Phi_{1}\right)\left(\Phi_{1}^{\dagger} \Phi_{2}\right)\right] \\
& +\lambda_{7}\left[\left(\Phi_{2}^{\dagger} \Phi_{2}\right)\left(\Phi_{2}^{\dagger} \Phi_{1}\right)+\left(\Phi_{2}^{\dagger} \Phi_{2}\right)\left(\Phi_{1}^{\dagger} \Phi_{2}\right)\right],
\end{aligned}
$$

where the scalar self-couplings are denoted by $\lambda_{1}-\lambda_{7}$. All calculations in the present paper are carried in the Landau gauge. This is merely a convenient choice, since many diagrams vanish in this gauge. We emphasize that the parameters of the effective Lagrangians are gauge fixing independent.

\section{Dimensional Reduction}

In this section we carry out the dimensional reduction step for the Two Higgs Doublet Model.

The fields in the effective Lagrangian are identified (up to normalizations) with the zerofrequency modes of the fields in the full theory. If the fields in the effective theory are denoted by $\Phi_{1}^{\prime}, \Phi_{2}^{\prime}, A_{i}^{\prime}$ and $A_{0}^{a \prime}$, we can schematically write at leading order

$$
\Phi_{i}^{\prime}(\mathbf{x}) \approx \sqrt{T} \int_{0}^{\beta} d \tau \Phi_{i}(\mathbf{x}, \tau), \quad A_{i}^{a \prime}(\mathbf{x}) \approx \sqrt{T} \int_{0}^{\beta} d \tau A_{i}^{a}(\mathbf{x}, \tau), \quad A_{0}^{a \prime}(\mathbf{x}) \approx \sqrt{T} \int_{0}^{\beta} d \tau A_{0}^{a}(\mathbf{x}, \tau)
$$


The effective Lagrangian consists of all terms which can built out of the fields $\Phi_{1}^{\prime}, \Phi_{2}^{\prime}, A_{i}^{\prime}$ and $A_{0}^{a \prime}$ and which satisfy the symmetries present at high temperature. Examples of symmetries are threedimensional gauge invariance and an $O(3)$ symmetry for the field $A_{0}^{a \prime}$. The effective Lagrangian then reads

$$
\begin{aligned}
\mathcal{L}_{\mathrm{eff}}^{\prime}= & \frac{1}{4} G_{i j}^{\prime} G_{i j}^{\prime}+\left(D_{i} \Phi_{1}^{\prime}\right)^{\dagger}\left(D_{i} \Phi_{1}^{\prime}\right)+\left(D_{i} \Phi_{2}^{\prime}\right)^{\dagger}\left(D_{i} \Phi_{2}^{\prime}\right)+M_{1}^{2}(\mu) \Phi_{1}^{\prime \dagger} \Phi_{1}^{\prime}+M_{2}^{2}(\mu) \Phi_{2}^{\prime \dagger} \Phi_{2}^{\prime} \\
& +M_{3}^{2}\left(\Phi_{1}^{\dagger} \Phi_{2}+\Phi_{2}^{\dagger} \Phi_{1}\right)+V\left(\Phi_{1}^{\prime}, \Phi_{2}^{\prime}\right)+\frac{1}{2}\left(D_{i} A_{0}^{a \prime}\right)^{2}+\frac{1}{2} m_{E}^{2}(\mu)\left(A_{0}^{a \prime}\right)^{2} \\
& +\frac{1}{24} \Lambda_{E}(\mu)\left(A_{0}^{a \prime} A_{0}^{a \prime}\right)^{2}+h_{E}^{2}(\mu) \Phi_{1}^{\prime \dagger} \Phi_{1}^{\prime} A_{0}^{a \prime} A_{0}^{a \prime}+h_{E}^{2}(\mu) \Phi_{2}^{\prime \dagger} \Phi_{2}^{\prime} A_{0}^{a \prime} A_{0}^{a \prime}+\delta \mathcal{L}_{\mathrm{eff}}^{\prime} .
\end{aligned}
$$

Here, we have explicitly written the superrenormalizable part of the Lagrangian, while $\delta \mathcal{L}_{\text {eff }}^{\prime}$ represents all higher order operators consistent with the symmetries of the Lagrangian. The gauge coupling is denoted by $g_{\mathrm{E}}^{2}(\mu)$, the quartic coupling constants in the potential $V\left(\Phi_{1}^{\prime}, \Phi_{2}^{\prime}\right)$ are denoted by $\Lambda_{i}(\mu)$, and $D_{i} A_{0}^{a}=\left(\partial_{i}+g_{E} \epsilon^{a b c} A_{i}^{b}\right) A_{0}^{c}$. The parameters of $\mathcal{L}_{\text {eff }}^{\prime}$ encode the physics at the scale $T$ and are called short-distance coefficients. The coefficients of the effective Lagrangian are determined by calculating static correlators in the full theory and calculating the corresponding correlators in the effective theory and require that they be equal at distances $R \gg 1 / T[27-28]$.

The matching procedure is complicated by the breakdown of the simple relations (5). Beyond leading order we must allow for short-distance coefficients multiplying the fields in the effective theory. At the one-loop level (next-to-leading order), the short-distance coefficients are given by the momentum dependent part of the two-point functions, and are associated with field renormalizations in the underlying theory. These parameters are called field normalization constants, and are denoted by $\Sigma^{(1) \prime}(0), \Pi_{00}^{(1) \prime}$ and $\Pi^{(1) \prime}$ for the Higgs fields, the timelike component of the gauge field and the spatial components of the gauge field, respectively. The relation between the fundamental scalar field $\Phi_{i}$ and the scalar field $\Phi_{i}^{\prime}$ in the effective theory can then schematically be written as

$$
\left[1-\Sigma^{(1) \prime}(0)\right]^{1 / 2} \Phi_{i}^{\prime}(\mathbf{x}) \approx \sqrt{T} \int_{0}^{\beta} d \tau \Phi_{i}(\mathbf{x}, \tau)
$$

and similarly for the other fields. The above remarks also apply when we consider the two effective three-dimensional field theories in the next section.

The field normalization constants have been calculated and listed by Kajantie et al. in [31] for the Standard Model with $N$ Higgs doublets. For $N=2$, the results are

$$
\Sigma_{1}^{\prime}(0)=-\frac{9 g^{2}}{64 \pi^{2}} L_{b}, \quad \Pi_{00}^{(1) \prime}(0)=-\frac{g^{2}}{16 \pi^{2}}\left[4 L_{b}-\frac{10}{3}\right], \quad \Pi^{(1) \prime}(0)=-\frac{g^{2}}{16 \pi^{2}}\left[4 L_{b}+\frac{2}{3}\right] .
$$

The coupling constants of the scalar fields have been calculated by Losada in [34] at the one-loop level. We list the results here for completeness.

$$
\Lambda_{1}(\mu)=\lambda_{1} T-T\left[12 \lambda_{1}^{2}+\lambda_{3}^{2}+\lambda_{3} \lambda_{4}+\frac{1}{2} \lambda_{4}^{2}+2 \lambda_{5}^{2}+6 \lambda_{6}^{2}-\frac{9}{2} \lambda_{1} g^{2}+\frac{9}{16} g^{4}\right] \frac{L_{b}}{16 \pi^{2}}+\frac{3}{8} \frac{g^{4} T}{16 \pi^{2}},(9)
$$




$$
\begin{aligned}
\Lambda_{2}(\mu)= & \lambda_{2} T-T\left[12 \lambda_{2}^{2}+\lambda_{3}^{2}+\lambda_{3} \lambda_{4}+\frac{1}{2} \lambda_{4}^{2}+2 \lambda_{5}^{2}+6 \lambda_{7}^{2}-\frac{9}{2} \lambda_{2} g^{2}+\frac{9}{16} g^{4}\right] \frac{L_{b}}{16 \pi^{2}}+\frac{3}{8} \frac{g^{4} T}{16 \pi^{2}}(10) \\
\Lambda_{3}(\mu)= & \lambda_{3} T-T\left[6 \lambda_{1} \lambda_{3}+2 \lambda_{1} \lambda_{4}+6 \lambda_{2} \lambda_{3}+2 \lambda_{2} \lambda_{4}+2 \lambda_{3}^{2}+\lambda_{4}^{2}+4 \lambda_{5}^{2}+2 \lambda_{6}^{2}+8 \lambda_{6} \lambda_{7}\right. \\
& \left.+2 \lambda_{7}^{2}-\frac{9}{2} \lambda_{3} g^{2}+\frac{9}{8} g^{4}\right] \frac{L_{b}}{16 \pi^{2}}+\frac{3}{4} \frac{g^{4} T}{16 \pi^{2}} \\
\Lambda_{4}(\mu)= & \lambda_{4} T-T\left[2 \lambda_{1} \lambda_{4}+2 \lambda_{2} \lambda_{4}+4 \lambda_{3} \lambda_{4}+2 \lambda_{4}^{2}+32 \lambda_{5}^{2}+5 \lambda_{6}^{2}+2 \lambda_{6} \lambda_{7}+5 \lambda_{7}^{2}\right. \\
& \left.-\frac{9}{2} \lambda_{4} g^{2}\right] \frac{L_{b}}{16 \pi^{2}} \\
\Lambda_{5}(\mu)= & \lambda_{5} T-T\left[4 \lambda_{1} \lambda_{5}+4 \lambda_{2} \lambda_{5}+8 \lambda_{3} \lambda_{5}+12 \lambda_{4} \lambda_{5}+5 \lambda_{6}^{2}+2 \lambda_{6} \lambda_{7}+5 \lambda_{7}^{2}\right. \\
& \left.-\frac{9}{2} \lambda_{5} g^{2}\right] \frac{L_{b}}{16 \pi^{2}} \\
\Lambda_{6}(\mu)= & \lambda_{6} T-T\left[12 \lambda_{1} \lambda_{6}+3 \lambda_{3} \lambda_{6}+3 \lambda_{3} \lambda_{7}+4 \lambda_{4} \lambda_{6}+2 \lambda_{4} \lambda_{7}+10 \lambda_{5} \lambda_{6}+2 \lambda_{5} \lambda_{7}\right. \\
& \left.-\frac{9}{2} \lambda_{6} g^{2}\right] \frac{L_{b}}{16 \pi^{2}} \\
& \left.\quad-\frac{9}{2} \lambda_{7} g^{2}\right] \frac{L_{b}}{16 \pi^{2}}
\end{aligned}
$$

The coupling constants $g_{E}^{2}(\mu), h_{E}^{2}(\mu)$ and $\Lambda_{E}^{4}(\mu)$ have been computed in e.g. Refs. 31, 34]:

$$
\begin{aligned}
g_{E}^{2}(\mu) & =g^{2} T\left[1+\frac{g^{2}}{16 \pi^{2}}\left(7 L_{b}+\frac{2}{3}\right)\right], \\
h_{E}^{2}(\mu) & =\frac{1}{4} g^{2} T\left[1+\frac{g^{2}}{16 \pi^{2}}\left(7 L_{b}+\frac{49}{6}\right)+\frac{3 \lambda_{1}}{4 \pi^{2}}+\frac{\lambda_{3}}{4 \pi^{2}}+\frac{\lambda_{4}}{8 \pi^{2}}\right], \\
\Lambda_{E}(\mu) & =\frac{3 g^{4} T}{8 \pi^{2}} .
\end{aligned}
$$

The coupling constants are all renormalization group invariant to this order, which can be verified by using the renormaliztion group equations. This property holds to all orders in perturbation theory if the effective Lagrangian is restricted to superrenormalizable terms.

The scalar mass parameters have been computed in the one-loop approximation by Losada in Ref. [34. Here, we present results for the mass parameters to two-loop order:

$$
\begin{aligned}
M_{1}^{2}(\mu)= & m_{1}^{2}-\left[6 m_{1}^{2} \lambda_{1}+2 m_{2}^{2} \lambda_{3}+m_{2}^{2} \lambda_{4}+6 m_{3}^{2} \lambda_{6}-\frac{9}{4} m_{1}^{2} g^{2}\right] \frac{L_{b}}{16 \pi^{2}} \\
& +\left[6 \Lambda_{1}+2 \Lambda_{3}+\Lambda_{4}+\frac{9}{4} g_{E}^{2}\right] \frac{T^{2}}{12}+\frac{T^{2}}{16 \pi^{2}}\left[\frac{3}{4} \lambda_{1} g^{2}+\frac{1}{4} \lambda_{3} g^{2}+\frac{1}{8} \lambda_{4} g^{2}+\frac{45}{32} g^{4}\right] \\
& -\frac{T^{2}}{16 \pi^{2}}\left[12 \lambda_{1}^{2}+2 \lambda_{3}^{2}+2 \lambda_{3} \lambda_{4}+2 \lambda_{4}^{2}+12 \lambda_{5}^{2}+9 \lambda_{6}^{2}+3 \lambda_{7}^{2}-9 \lambda_{1} g^{2}-3 \lambda_{3} g^{2}\right.
\end{aligned}
$$




$$
\begin{aligned}
& \left.-\frac{3}{2} \lambda_{4} g^{2}-\frac{75}{16} g^{4}\right]\left[\ln \frac{3 T}{\mu}+c\right], \\
M_{2}^{2}(\mu)= & m_{2}^{2}-\left[6 m_{2}^{2} \lambda_{2}+2 m_{1}^{2} \lambda_{3}+m_{1}^{2} \lambda_{4}+6 m_{3}^{2} \lambda_{7}-\frac{9}{4} g^{2} m_{2}^{2}\right] \frac{L_{b}}{16 \pi^{2}} \\
& +\left(6 \Lambda_{2}+2 \Lambda_{3}+\Lambda_{4}+\frac{9}{4} g_{E}^{2}\right) \frac{T^{2}}{12}+\frac{T^{2}}{16 \pi^{2}}\left[\frac{3}{4} \lambda_{2} g^{2}+\frac{1}{4} \lambda_{3} g^{2}+\frac{1}{8} \lambda_{4} g^{2}+\frac{45}{32} g^{4}\right] \\
& -\frac{T^{2}}{16 \pi^{2}}\left[12 \lambda_{2}^{2}+2 \lambda_{3}^{2}+2 \lambda_{3} \lambda_{4}+2 \lambda_{4}^{2}+12 \lambda_{5}^{2}+3 \lambda_{6}^{2}+9 \lambda_{7}^{2}-9 \lambda_{2} g^{2}-3 \lambda_{3} g^{2}\right. \\
& \left.-\frac{3}{2} \lambda_{4} g^{2}-\frac{75}{16} g^{4}\right]\left[\ln \frac{3 T}{\mu}+c\right], \\
M_{3}^{2}(\mu)= & m_{3}^{2}-\left[m_{3}^{2} \lambda_{3}+2 m_{3}^{2} \lambda_{4}+6 m_{3}^{2} \lambda_{5}+3 m_{1}^{2} \lambda_{6}+3 m_{2}^{2} \lambda_{7}-\frac{9}{4} g^{2} m_{3}^{2}\right] \frac{L_{b}}{16 \pi^{2}} \\
& +\left(\Lambda_{6}+\Lambda_{7}\right) \frac{T^{2}}{4}+\frac{T^{2}}{16 \pi^{2}}\left[\frac{3}{8} \lambda_{6} g^{2}+\frac{3}{8} \lambda_{7} g^{2}\right] \\
& -\frac{T^{2}}{16 \pi^{2}}\left[6 \lambda_{1} \lambda_{6}+6 \lambda_{2} \lambda_{7}+3 \lambda_{3} \lambda_{6}+3 \lambda_{3} \lambda_{7}+3 \lambda_{4} \lambda_{6}+3 \lambda_{4} \lambda_{7}+6 \lambda_{5} \lambda_{6}+6 \lambda_{5} \lambda_{7}\right. \\
& \left.-\frac{9}{2} \lambda_{6} g^{2}-\frac{9}{2} \lambda_{7} g^{2}\right]\left[\ln \frac{3 T}{\mu}+c\right] \cdot
\end{aligned}
$$

Here, $c$ is the constant 27

$$
c=\frac{1}{2}\left[\ln \frac{8 \pi}{9}+\frac{\zeta^{\prime}(2)}{\zeta(2)}-2 \gamma_{E}\right] \approx-0.348725 .
$$

Note that we have written our mass parameters in terms of the renormalization group invariant couplings of the $3 d$ theory. The remaining dependence on $\mu$ reveals that scalar mass parameters depend explicitly on the scale $\mu$. This dependence on the scale $\mu$ is canceled by the scale dependence arising from calculations in the effective theory.

The Debye mass is normally needed in the one-loop approximation 31

$$
m_{E}^{2}(\mu)=g^{2} T^{2} .
$$

There is no dependence on $\mu$ at leading order in $g^{2}$.

\section{$4 \quad$ Integrating out $A_{0}^{a \prime}$}

The next step is to integrate out the adjoint scalar triplet $A_{0}^{a \prime}$. This is carried out by calculating correlators in the two theories at distances $R \gg 1 / g T$ and require that they be the same [27-28]. The parameters in the effective theory encode the physics on the scales $T$ and $g T$ and are called 
middle-distance coefficients. Before doing this, however, we must determine the masses of the scalar doublets near the phase transition. This is done by constructing the scalar mass matrix and finding the temperatures at which it has zero eigenvalues. The higher of these temperatures is close to $T_{c}$, where the phase transition takes place and the corresponding eigenvector (Higgs doublet) has a mass of order $g^{2} T$. The mass of the second scalar multiplet (after diagonalization) is determined near $T_{c}$ and it is found that it is generally of order $g T$ and it should be integrated

out together with $A_{0}^{a \prime}$ [31. Only with fine-tuning of the parameters in the 2HDM, is it possible to obtain a mass of order $g^{2} T$ [31]. In this case it must be kept in the second effective Lagrangian. Both cases are considered below. The diagonalization modifies the parameters of (6) and the relations between the old and new parameters can be found in [32, 34]. In the following it is the rotated parameters of (6) that appear in the formulas.

\subsection{One Heavy Higgs and one Light Higgs}

In this subsection we consider the case where one of the Higgs fields (denoted by $\Phi_{2}^{\prime}$ ) is heavy and has a mass of order $g T$. Hence, we integrate out this field together with the adjoint Higgs field $A_{0}^{a}$. The second effective field theory is then $S U(2)+$ one Higgs doublet with higher order operators satisfying the symmetries. The Lagrangian reads

$$
\tilde{\mathcal{L}}_{\text {eff }}=\frac{1}{4} \tilde{G}_{i j} \tilde{G}_{i j}+\tilde{M}_{1}^{2}(\mu) \tilde{\Phi}_{1}^{\dagger} \tilde{\Phi}_{1}+\left(D_{i} \tilde{\Phi}_{1}\right)^{\dagger}\left(D_{i} \tilde{\Phi}_{1}\right)+\tilde{\Lambda}_{1}(\mu)\left(\tilde{\Phi}_{1}^{\dagger} \tilde{\Phi}_{1}\right)^{2}+\delta \tilde{\mathcal{L}}_{\text {eff }}
$$

The gauge coupling is denoted by $g_{M}^{2}(\mu)$.

The field renormalization constant for the scalar fields vanish in the one-loop approximation, since there are no momentum dependent one-loop diagrams with internal $A_{0}^{a /}$ 's. Thus

$$
\tilde{\Phi}_{i}(\mu) \approx \Phi_{i}^{\prime}(\mu)
$$

This is in contrast with the gauge fields, since there is a momentum dependent one-loop diagram with $A_{0}^{a \prime}$ on the internal lines and $A_{i}^{a \prime}$ on the external lines. The result is [32]

$$
\tilde{A}_{i}^{a} \approx A_{i}^{a \prime}\left[1+\frac{g_{E}^{2}}{24 \pi m_{E}}+\frac{g_{E}^{2}}{48 \pi M_{2}}\right]^{1 / 2}
$$

The results for the coupling constants can be found in [32, 34]:

$$
\begin{aligned}
\tilde{\Lambda}_{1}(\mu) & =\Lambda_{1}(\mu)-\frac{1}{16 \pi M_{2}}\left[2 \Lambda_{3}^{2}+2 \Lambda_{3} \Lambda_{4}+\Lambda_{4}^{2}+4 \Lambda_{5}^{2}+24 \Lambda_{6}^{2}-24 \Lambda_{6} \Lambda_{7}\right]-\frac{3 h_{E}^{2}}{8 \pi m_{E}} \\
g_{M}^{2}(\mu) & =g_{E}^{2}(\mu)\left[1-\frac{g_{E}^{2}}{24 \pi m_{E}}-\frac{g_{E}^{2}}{48 \pi M_{2}}\right] .
\end{aligned}
$$


The mass parameter has previously been computed by Losada [34] at one-loop. The result in the two-loop approximation is:

$$
\begin{aligned}
\tilde{M}_{1}^{2}(\mu)= & M_{1}^{2}(\mu)-\frac{1}{4 \pi}\left[\left(2 \Lambda_{3}+\Lambda_{4}\right) M_{2}+3 h_{E}^{2} m_{E}\right]+\frac{3}{16 \pi^{2}}\left[2 \Lambda_{2} \Lambda_{3}+\Lambda_{2} \Lambda_{4}+6 \Lambda_{6} \Lambda_{7}\right] \\
& -\frac{9}{16 \pi^{2}} \Lambda_{6}^{2}\left[\ln \frac{\mu}{M_{2}}+\frac{1}{2}\right]-\frac{3}{16 \pi^{2}} \Lambda_{7}^{2}\left[\ln \frac{\mu}{3 M_{2}}+\frac{1}{2}\right] \\
& -\frac{1}{16 \pi^{2}}\left[\left(2 \Lambda_{3}^{2}+2 \Lambda_{3} \Lambda_{4}+2 \Lambda_{4}^{2}+12 \Lambda_{5}^{2}-3 \Lambda_{3} g_{E}^{2}-\frac{3}{2} \Lambda_{4} g_{E}^{2}\right) \ln \frac{\mu}{2 M_{2}}\right. \\
& \left.+\Lambda_{3}^{2}+\Lambda_{3} \Lambda_{4}+\Lambda_{2}^{4}+6 \Lambda_{5}^{2}-\frac{3}{4} \Lambda_{3} g_{E}^{2}-\frac{3}{8} \Lambda_{4} g_{E}^{2}\right] \\
& -\frac{1}{16 \pi^{2}}\left[\left(6 h_{E}^{4}-12 h_{E}^{2} g_{E}^{2}+\frac{3}{4} g_{E}^{4}\right) \ln \frac{\mu}{2 m_{E}}+3 h_{E}^{4}-3 h_{E}^{2} g_{E}^{2}\right] \\
& +\frac{1}{16 \pi^{2}}\left[6 h_{E}^{4} \frac{M_{2}}{m_{E}}+3 \Lambda_{3} h_{E}^{2} \frac{m_{E}}{M_{2}}+\frac{3}{2} \Lambda_{4} h_{E}^{2} \frac{m_{E}}{M_{2}}\right] .
\end{aligned}
$$

\subsection{Two Light Higgs Doublets}

In this subsection we consider the other scenario when both Higgs fields have masses of order $g^{2} T$. The effective Lagrangian is now a three-dimensional 2HDM with additional higher order operators which satisfy the symmetries at high temperature:

$$
\begin{aligned}
\tilde{\mathcal{L}}_{\mathrm{eff}}= & \frac{1}{4} \tilde{G}_{i j} \tilde{G}_{i j}+\left(D_{i} \tilde{\Phi}_{1}\right)^{\dagger}\left(D_{i} \tilde{\Phi}_{1}\right)+\left(D_{i} \tilde{\Phi}_{2}\right)^{\dagger}\left(D_{i} \tilde{\Phi}_{2}\right)+\tilde{M}_{1}^{2}(\mu) \tilde{\Phi}_{1}^{\dagger} \tilde{\Phi}_{1}+\tilde{M}_{2}^{2}(\mu) \tilde{\Phi}_{2}^{\dagger} \tilde{\Phi}_{2} \\
& +\tilde{M}_{3}^{2}(\mu)\left(\tilde{\Phi}_{1}^{\dagger} \tilde{\Phi}_{2}+\tilde{\Phi}_{2}^{\dagger} \tilde{\Phi}_{1}\right)+V\left(\tilde{\Phi}_{1}, \tilde{\Phi}_{2}\right)+\delta \tilde{\mathcal{L}}_{\mathrm{eff}} .
\end{aligned}
$$

The scalar couplings are denoted by $\tilde{\Lambda}_{i}$ and the gauge coupling by $g_{M}^{2}(\mu)$.

Again the scalar fields are not renormalized by integrating out the $A_{0}^{a \prime}$ fields and so (25) also holds in the present case. This is in contrast with the gauge fields, since there is a trilinear coupling between $A_{0}^{a \prime}$ and $A_{i}^{a \prime}$ :

$$
\tilde{A}_{i}^{a} \approx A_{i}^{a \prime}\left[1+\frac{g_{E}^{2}}{24 \pi m_{E}}\right]^{1 / 2} .
$$

The scalar couplings $\tilde{\Lambda}_{1}(\mu)-\tilde{\Lambda}_{3}(\mu)$ get modified by the integrating out $A_{0}^{a}$ :

$$
\tilde{\Lambda}_{1}(\mu)=\Lambda_{1}(\mu)-\frac{3 h_{E}^{4}}{8 \pi m_{E}}, \quad \tilde{\Lambda}_{2}(\mu)=\Lambda_{2}(\mu)-\frac{3 h_{E}^{4}}{8 \pi m_{E}}, \quad \tilde{\Lambda}_{3}(\mu)=\Lambda_{3}(\mu)-\frac{3 h_{E}^{4}}{4 \pi m_{E}} .
$$

The other coupling constants, $\tilde{\Lambda}_{4}(\mu)-\tilde{\Lambda}_{7}(\mu)$, are not modified in this step. The gauge coupling reads

$$
g_{M}^{2}(\mu)=g_{E}^{2}(\mu)\left[1-\frac{g_{E}^{2}}{24 \pi m_{E}}\right] .
$$


The expression for the mass parameters at the two-loop level are

$$
\begin{aligned}
& \tilde{M}_{1}^{2}(\mu)=M_{1}^{2}(\mu)-\frac{3 h_{E}^{2} m_{E}}{4 \pi}-\frac{1}{16 \pi^{2}}\left[\left(6 h_{E}^{4}-12 h_{E}^{2} g_{E}^{2}+\frac{3}{4} g_{E}^{4}\right) \ln \frac{\mu}{2 m_{E}}+3 h_{E}^{4}-3 h_{E}^{2} g_{E}^{2}\right] \\
& \tilde{M}_{2}^{2}(\mu)=M_{2}^{2}(\mu)-\frac{3 h_{E}^{2} m_{E}}{4 \pi}-\frac{1}{16 \pi^{2}}\left[\left(6 h_{E}^{4}-12 h_{E}^{2} g_{E}^{2}+\frac{3}{4} g_{E}^{4}\right) \ln \frac{\mu}{2 m_{E}}+3 h_{E}^{4}-3 h_{E}^{2} g_{E}^{2}\right] \\
& \tilde{M}_{3}(\mu)=M_{3}(\mu) .
\end{aligned}
$$

\section{Summary}

In the present paper I have applied the effective field theory methods developed in Refs. [27-28] to the 2HDM. I have exploited the fact that there are three well separated momentum scales and constructed a sequence of two effective three-dimensional field theories. The parameters in the final effective Lagrangian have previosuly been calculated in the one-loop approximation [34]. The two-loop results presented here are new.

The resulting field theory can be used for investigating several aspects of the phase transition in the Two Higgs Doublet Model. This includes in particular the strength of the phase transition, and also the sphaleron rate immediately after the completion of the phase transition.

\section{A Notation and Conventions}

Throughout the work we use the imaginary time formalism, where the four-momentum is $P=$ $\left(p_{0}, \mathbf{p}\right)$ with $P^{2}=p_{0}^{2}+\mathbf{p}^{2}$. The Euclidean energy takes on discrete values, $p_{0}=2 n \pi T$ for bosons. Dimensional regularization is used to regularize both infrared and ultraviolet divergences by working in $d=4-2 \epsilon$ dimensions, and we apply the $\overline{M S}$ renormalization scheme. We shall use the following notations for the sum-integrals that appear

$$
\oiint_{P} f(P) \equiv\left(\frac{e^{\gamma_{E}} \mu^{2}}{4 \pi}\right)^{\epsilon} T \sum_{p_{0}=2 \pi n T} \int \frac{d^{3-2 \epsilon} p}{(2 \pi)^{3-2 \epsilon}} f(P) \text {. }
$$

The one-loop sum-integrals needed in this work have been calculated in e.g. Refs. [8,28,31]. We list them here for the convenience of the reader:

$$
\oiint_{P} \frac{1}{P^{2}}=\frac{T^{2}}{12}\left[1+\epsilon l_{\epsilon}\right],
$$




$$
\begin{aligned}
& \sum_{P} \frac{1}{\left(P^{2}\right)^{2}}=\frac{1}{16 \pi^{2}}\left[\frac{1}{\epsilon}+L_{b}+\mathcal{O}(\epsilon)\right] \text {, } \\
& \xi_{P} \frac{P_{0}^{2}}{\left(P^{2}\right)^{2}}=-\frac{T^{2}}{24}\left[1+\epsilon\left(l_{\epsilon}-2\right)\right] \text {, } \\
& \oiint_{P} \frac{P_{0}^{2}}{\left(P^{2}\right)^{3}}=\frac{1}{64 \pi^{2}}\left[\frac{1}{\epsilon}+L_{b}+2+\mathcal{O}(\epsilon)\right] \text {, } \\
& \mathcal{F}_{P} \frac{P_{0}^{4}}{\left(P^{2}\right)^{4}}=\frac{1}{128 \pi^{2}}\left[\frac{1}{\epsilon}+L_{b}+\frac{8}{3}+\mathcal{O}(\epsilon)\right] \text {. }
\end{aligned}
$$

Here

$$
L_{b}=2 \ln \frac{\mu}{4 \pi T}+2 \gamma_{E}, \quad l_{\epsilon}=2 \ln \frac{\mu}{T}+2 \gamma_{E}-2 \ln 2-2 \frac{\zeta^{\prime}(2)}{\zeta(2)} .
$$

Moreover, $\gamma_{E}$ is the Euler-Mascharoni constant and $\zeta(x)$ is the Riemann Zeta function.

In the calculation of the mass parameters, we also need the fact that two-loop setting sun diagram is zero 282:

$$
\oiint_{P Q} \frac{1}{P^{2} Q^{2}(P+Q)^{2}}=0 .
$$

In the effective three-dimensional theory we also use dimensional regularization in $3-2 \epsilon$ dimensions to regularize infrared and ultraviolet divergences. In analogy with (A.1), we define

$$
\int_{p} f(p) \equiv\left(\frac{e^{\gamma_{E}} \mu^{2}}{4 \pi}\right)^{\epsilon} \int \frac{d^{3-2 \epsilon} p}{(2 \pi)^{3-2 \epsilon}} f(p) .
$$

Again $\mu$ coincides with the renormalization scale in the modified minimal subtraction renormalization scheme.

The one-loop and two-loop integrals needed are

$$
\begin{aligned}
\int_{p} \frac{1}{p^{2}+m^{2}} & =-\frac{m}{4 \pi}[1+\mathcal{O}(\epsilon)], \\
\int_{p} \frac{1}{\left(p^{2}+m_{1}^{2}\right)\left(p^{2}+m_{2}^{2}\right)}= & \frac{1}{4 \pi\left(m_{1}+m_{2}\right)}[1+\mathcal{O}(\epsilon)], \\
\int_{p q} \frac{1}{\left(p^{2}+m_{1}^{2}\right)\left(q^{2}+m_{2}^{2}\right)\left[(\mathbf{p}-\mathbf{q})^{2}+m_{3}^{2}\right]}= & \frac{1}{16 \pi^{2}}\left[\frac{1}{4 \epsilon}+\frac{1}{2}\right. \\
& \left.+\ln \frac{\mu}{m_{1}+m_{2}+m_{3}}+\mathcal{O}(\epsilon)\right], \\
\int_{p q} \frac{1}{\left(p^{2}+m^{2}\right)\left(q^{2}+m^{2}\right)^{2}(\mathbf{p}-\mathbf{q})^{2}}= & \frac{1}{16 \pi^{2} m^{2}}\left[\frac{1}{4}+\mathcal{O}(\epsilon)\right], \\
\int_{p q} \frac{1}{\left(p^{2}+m^{2}\right)\left(q^{2}+m^{2}\right)(\mathbf{p}-\mathbf{q})^{4}}= & \frac{1}{16 \pi^{2} m^{2}}\left[-\frac{1}{8}+\mathcal{O}(\epsilon)\right] .
\end{aligned}
$$

These integrals have been computed by several authors, e.g. in Refs. [8, 28, 31. 


\section{B Matching Example}

In this appendix we explicitly show how the matching procedure is carried out by determining the mass parameter $M_{1}^{2}(\Lambda)$ to two-loop order.

We denote the static two-point function of the Higgs field in the full theory by $\Gamma_{\phi_{1}, \phi_{1}}^{(2)}(\mathbf{k})$, and the static two-point function in the effective theory by $\Gamma_{\phi_{1}^{\prime}, \phi_{1}^{\prime}}^{(2)}(\mathbf{k})$. The corresponding self-energies are denoted by $\Sigma(\mathbf{k})$ and $\tilde{\Sigma}(\mathbf{k})$. Finally, the n'th order contribution to the self-energies in the loop expansion are denoted by $\Sigma^{(n)}(\mathbf{k})$ and $\tilde{\Sigma}^{(n)}(\mathbf{k})$.

The self-energies can be expanded in powers of the external momentum $\mathbf{k}$ and so we can write the two-point functions as

$$
\begin{aligned}
& \Gamma_{\phi_{1}, \phi_{1}}^{(2)}(\mathbf{k})=k^{2}+m_{1}^{2}+\Sigma^{(1)}(0)+k^{2} \Sigma^{(1) \prime}(0)+\Sigma^{(2)}(0), \\
& \Gamma_{\phi_{1}^{\prime}, \phi_{1}^{\prime}}^{(2)}(\mathbf{k})=k^{2}+M_{1}^{2}(\mu)+\tilde{\Sigma}^{(1)}(0)+k^{2} \tilde{\Sigma}^{(1) \prime}(0)+\tilde{\Sigma}^{(2)}(0)+\delta M_{1}^{2} .
\end{aligned}
$$

Here, we have added a mass counterterm, which is associated with mass renormalization. The mass parameter is then determined by matching these two-point functions, and by taking the field normalization constant into account, we can write the matching equation as

$$
\Gamma_{\phi_{1}, \phi_{1}}^{(2)}(\mathbf{k})=\left[1+\Sigma^{(1) \prime}(0)\right] \Gamma_{\phi_{1}^{\prime}, \phi_{1}^{\prime}}^{(2)}(\mathbf{k}) .
$$

Since the external momentum $\mathbf{k}$ provides the only mass scale in the loop integrals contributing to the self-energy of the effective theory, they all vanish in dimensional regularization. The matching equation $(\mathbb{B} .3)$ can then be rewritten as

$$
M_{1}^{2}(\mu)=m_{1}^{2}\left[1-\Sigma^{(1) \prime}(0)\right]+\Sigma^{(1)}(0)\left[1-\Sigma^{(1) \prime}(0)\right]+\Sigma^{(2)}(0)-\delta M_{1}^{2} .
$$

The self-energy at one-loop order in the full theory reads:

$$
\begin{aligned}
\Sigma^{(1)}(\mathbf{k})= & -\left[6 m_{1}^{2} \lambda_{1}+2 m_{2}^{2} \lambda_{3}+m_{2}^{2} \lambda_{4}+6 m_{3}^{2} \lambda_{6}\right] \mathcal{F}_{P} \frac{1}{P^{4}} \\
& +\left[6 \lambda_{1}+2 \lambda_{3}+\lambda_{4}+\frac{3}{4}(d-1) g^{2}\right] \oiint_{P} \frac{1}{P^{2}} \\
& -3 g^{2} \oint_{P} \frac{k^{2}}{P^{2}(P+K)^{2}}+3 g^{2} \oint_{P} \frac{(\mathbf{p k})^{2}}{P^{4}(P+K)^{2}} .
\end{aligned}
$$

Expanding in powers of the external momentum $\mathbf{k}$ gives

$$
\begin{aligned}
\Sigma^{(1)}(\mathbf{k})= & -\left[6 m_{1}^{2} \lambda_{1}+2 m_{2}^{2} \lambda_{3}+m_{2}^{2} \lambda_{4}+6 m_{3}^{2} \lambda_{6}\right] \oiint_{P} \frac{1}{P^{4}} \\
& +\left[6 \lambda_{1}+2 \lambda_{3}+\lambda_{4}+\frac{3}{4}(d-1) g^{2}\right] \oiint_{P} \frac{1}{P^{2}}-\frac{9}{4} g^{2} k^{2} \sum_{P} \frac{1}{P^{4}}+\mathcal{O}\left(k^{4} / T^{2}\right) .
\end{aligned}
$$


This implies

$$
\begin{aligned}
\Sigma^{(1)}(0)= & -\left[6 m_{1}^{2} \lambda_{1}+2 m_{2}^{2} \lambda_{3}+m_{2}^{2} \lambda_{4}+6 m_{3}^{2} \lambda_{6}\right] \oint_{P} \frac{1}{P^{4}} \\
& +\left[6 \lambda_{1}+2 \lambda_{3}+\lambda_{4}+\frac{3}{4}(d-1) g^{2}\right] \oiint_{P} \frac{1}{P^{2}}, \\
\Sigma^{(1) \prime}(0)= & -\frac{9}{4} g^{2} \oiint_{P} \frac{1}{P^{4}} .
\end{aligned}
$$

The two-loop contribution to the self-energy at zero external momentum is

$$
\begin{aligned}
\Sigma^{(2)}(0)= & -\left[36 \lambda_{1}^{2}+12 \lambda_{1} \lambda_{3}+6 \lambda_{1} \lambda_{4}+12 \lambda_{2} \lambda_{3}+6 \lambda_{2} \lambda_{4}+4 \lambda_{3}^{2}+4 \lambda_{3} \lambda_{4}+\lambda_{4}^{2}\right. \\
& +18 \lambda_{6}^{2}+18 \lambda_{6} \lambda_{7}+\frac{9}{2}(d-1) \lambda_{1} g^{2}+\frac{3}{2}(d-1) \lambda_{3} g^{2}+\frac{3}{4}(d-1) \lambda_{4} g^{2} \\
& \left.+\frac{1}{2}\left(3 d^{2}-9 d+6\right) g^{4}\right] \sum_{P Q} \frac{1}{P^{2} Q^{4}} .
\end{aligned}
$$

After renormalization of the mass parameter $m_{1}^{2}$ as well as the coupling constants, we are left with a pole in $\epsilon$. This pole is canceled by the mass renormalization counterterm, which is

$$
\begin{aligned}
\delta M_{1}^{2}= & {\left[12 \lambda_{1}^{2}+2 \lambda_{3}^{2}+2 \lambda_{3} \lambda_{4}+2 \lambda_{4}^{2}+12 \lambda_{5}^{2}+9 \lambda_{6}^{2}+3 \lambda_{7}^{2}-9 \lambda_{1} g^{2}-3 \lambda_{3} g^{2}\right.} \\
& \left.-\frac{3}{2} \lambda_{4} g^{2}-\frac{75}{16} g^{4}\right] \frac{1}{64 \pi^{2} \epsilon} .
\end{aligned}
$$

This is the result for the mass counterterm of the three-dimensional 2HDM at next-to-leading order in the coupling constants. The mass parameter $M_{1}^{2}(\mu)$ is then given by $(19)$.

This work was supported in part by a Faculty Development Grant from the Physics Department of the Ohio State University and by the Norwegian Research Council (project 124282/410).

\section{References}

[1] V. A. Kuzmin, V. A. Rubakov and M. E. Shaposhnikov, Phys. Lett. B155, 36, 1985.

[2] M. E. Shaposhnikov, Nucl. Phys. B287, 757, 1987.

[3] M. Trodden, hep-ph/9803479.

[4] A. G. Cohen, D. B. Kaplan and A. E. Nelson, Ann. Rev. Nucl. Part. Sci. 43, 27, 1994.

[5] K. Kajantie, M. Laine, K. Rummukainen and M. Shaposhnikov, Phys. Rev. Lett. 77, 2887, 1996. 
[6] J. Gunion, H. Haber, G. Kane and S. Dawson, The Higgs Hunter's Guide. Addison-Wesley, Redwood City, CA, 1990.

[7] M. E. Carrington, Phys. Rev D45, 2933, 1992.

[8] P. Arnold and O. Espinosa, Phys. Rev. D47, 3546, 1993.

[9] Z. Fodor and A. Hebecker, Nucl. Phys. B432, 127, 1994; W. Buchmuller, Z. Fodor and A. Hebecker, Nucl.Phys. B447, 317, 1995.

[10] A. I. Bocharev, S. V. Kuzmin and M. E. Shaposhnikov, Phys. Lett. B244, 275, 1990; Phys. Rev. D43, 369, 1991

[11] N. Turok and J. Zadrozny, Nucl. Phys. B369, 729, 1992.

[12] A .T. Davies, C.D. Froggatt, G. Jenkins and R.G. Moorhouse, Phys. Lett. B336, 464, 1994.

[13] J. M. Cline and K. Kainulainen, Phys. Rev. D54, 2451, 1996.

[14] J. M. Cline and P.-A. Lemieux, Phys. Rev. D55, 3873, 1997.

[15] F. Csikor, Z. Fodor, J. Hein, K. Jansen, A. Jaster and I. Montvay, Nucl. Phys. Proc. Suppl. 42, 569, 1995.

[16] Z. Fodor, J. Hein, K. Jansen, A. Jaster and I. Montvay, Nucl. Phys. B439, 147, 1995.

[17] Z. Fodor, J. Hein, K. Jansen, A. Jaster, I. Montvay and F. Csikor, Phys. Lett. B334, 405, 1994.

[18] J. March-Russell, Phys. Lett. B296, 364, 1992.

[19] M. Alford and J. March-Russell, Nucl. Phys. B417, 527, 1994.

[20] P. Arnold and L. G. Yaffe, Phys. Rev. D49, 3003, 1994.

[21] N. P. Landsman. Nucl. Phys. B322, 498, 1989.

[22] P. Ginsparg, Nucl. Phys. B170, 388, 1980.

[23] T. Appelquist and R. Pisarski, Phys. Rev. D23, 2305, 1981

[24] S. Nadkarni, Phys. Rev. D27, 917, 1983.

[25] G. P. Lepage, in From actions to answers, proceedings of the Theoretical Advanced Study Institute in Elementary Particle Physics, Boulder, Colorado, 1989, edited by T. De-Grand and D. Toussaint, World Scientific, 1989.

[26] D. J. Gross, R. D. Pisarski and L. G. Yaffe, Rev. Mod. Phys. 53, 43, 1981. 
[27] K. Farakos, K. Kajantie, K. Rummukainen, M.Shaposhnikov. Nucl. Phys. B425, 67, 1994.

[28] E. Braaten and A. Nieto, Phys. Rev. D51, 6990, 1995; Phys. Rev. D53, 3421, 1996.

[29] Kajantie, K. Rummukainen, M. Shaposhnikov, Nucl. Phys. B407, 356, 1993.

[30] A. Jakovac, K. Kajantie and A. Patkos, Phys. Rev. D49, 6810, 1994.

[31] K. Kajantie, M. Laine, K. Rummukainen, M. Shaposhnikov, Nucl. Phys. B458, 90, 1996.

[32] M. Laine, Nucl. Phys. B481, 43, 1996.

[33] J. M. Cline and K. Kainulainen, Nucl. Phys. B482, 73, 1996.

[34] M. Losada, Phys. Rev. D56, 2893, 1997.

[35] G. R. Farrar and M. Losada, Phys. Lett. B406, 60, 1997.

[36] D. Bödeker, P. John, M. Laine, and M. G. Schmidt, Nucl. Phys. B497, 387, 1997.

[37] A. Rajantie, Nucl. Phys. B501, 521, 1997.

[38] J. O. Andersen, Phys. Rev. D59, 065018, 1999.

[39] K. Kajantie, M. Karjalainen, M. Laine and J. Peisa, Nucl. Phys. B520, 345, 1998.

[40] K. Kajantie, K. Rummukainen and M. Shaposhnikov, Nucl. Phys. B407, 356 1993; K. Kajantie, M. Laine, K. Rummukainen and M. Shaposhnikov, Nucl. Phys. B466, 1891996.

[41] F. Karsch, T. Neuhaus, A. Patkós and J. Rank, Nucl. Phys. B474, 217, 1996.

[42] E.-M. Ilgenfritz, J. Kripfganz, H. Perlt and A. Schiller, Phys. Lett. B356, 561, 1995; M. Gütler, E.-M.Ilgenfritz, H. Perlt and A. Schiller, Nucl. Phys. B483, 383, 1997; M. Gütler, E.-M.Ilgenfritz and A. Schiller, Eur. Phys. J. C1, 363, 1998, Phys. Rev. D56, 3888, 1997.

[43] G. D. Moore and N. Turok, Phys. Rev. D55, 6538, 1997.

[44] O. Phillipsen, M. Teper and H. Wittig, Nucl. Phys. B469, 445, 1996; Nucl. Phys. B528, 379, 1998.

[45] K. Kajantie, M. Laine, K. Rummukainen and M. Shaposhnikov, Nucl. Phys. B493, 413, 1997.

[46] B. I. Halperin, T. C. Lubensky and S.-K. Ma, Phys. Rev. Lett. 32, 292, 1974.

[47] C. Dasgupta and B. I. Halperin, Phys. Rev. Lett. 47, 1556, 1981.

[48] J. Bartholomew, Phys. Rev. B28, 5378, 1983. 
[49] Y. Munehisa, Phys. Lett. B155, 159, 1985.

[50] P. Dimopoulos, K. Farakos and G. Kotsoumbas, Eur. Phys. J. C1, 711, 1998.

[51] M. Karjalainen and J. Peisa, Z. Phys. C76, 319, 1996.

[52] M. Karjalainen, M. Laine and J. Peisa, Nucl. Phys. Proc. Suppl. 53, 475, 1997.

[53] K. Kajantie, M. Karjalainen, M. Laine and J. Peisa, Phys. Rev. B57, 3011, 1998.

[54] M. Laine and K. Rummukainen, Phys. Rev. Lett. 80, 5259, 1998. Nucl. Phys. B535, 423, 1998. 